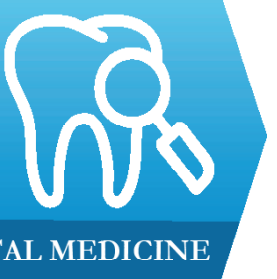

DENTAL MEDICINE

\title{
Role of Cytokeratin-7 in the pathogenesis of odontogenic cysts - an immunohistochemical study
}

Pulin Saluja $^{1}$, Manpreet Arora ${ }^{1}$, Aparna Dave ${ }^{1}$, Vishwa Prakash Shetty ${ }^{2}$, Charu Khurana ${ }^{3}$, Ajay Madan ${ }^{4}$, Radhika Rai ${ }^{1}$, Alpana Katiyar ${ }^{5}$

1) Oral Pathology Department, SGT Dental Collage, Hospital and Research Institute, Gurgaon, India

2) Oral Pathology Department, Lenora Institute of Dental Sciences, Rajahmundry, India

3) National Oral Health Program (NOHP), Centre for Dental Education and Research (CDER), All India Institute of Medical Sciences (AIIMS), New Delhi, India

4) General Surgery Department, Brahmashakti Sanjivani Hospital, Bahadurgarh, India

5) Pedodontics Department, Maitri College of Dentistry and Research, Chhattisgarh, India

DOI: $10.15386 / \mathrm{mpr}-1112$

Manuscript received: 25.07.2018

Accepted: 28.09.2018

Address for correspondence:

drcharukhurana@gmail.com

\begin{abstract}
Introduction. Odontogenic cysts are distinct entities and quite a common occurrence in the jaw bones. These are individual lesions which arise from the same odontogenic apparatus but with varying pathogenesis. Cytokeratins are integral components in tooth development and are expressed across the odontogenic tissues in physiological and pathological states.
\end{abstract}

Aim. To elucidate the role of cytokeratin-7 in the pathogenesis of odontogenic cysts by immunohistochemistry.

Method. Cytokeratin-7 (CK-7) was assessed in 39 cases of odontogenic lesions retrieved from the archival files which included 15 cases of dentigerous cysts (DC), 12 cases of odontogenic keratocysts (OKC) and 12 cases of radicular cysts (RC) and also 8 cases of control specimens.

Statistical analysis. Results obtained were statistically analyzed using chi-square test to assess the association between different odontogenic cysts used in this study and Cytokeration-7 staining. The difference was considered to be of statistical significance if the $\mathrm{p}$ value was $\leq 0.05$.

Results. CK7 expression was maximum in dentigerous cycts (66.66\%) followed by radicular cysts $(41.66 \%)$ and odontogenic keratocysts $(16.6 \%)$. On evaluation of staining and expression pattern, highest positivity is shown in dentigerous cysts and the positivity is seen in suprabasal $(60 \%)$ and superficial layers $(40 \%)$ whereas radicular cysts and odontogenic keratocysts showed positivity in superficial and spinous layers.

Conclusion. Cytokeratin-7 expression correlates with the degree of differentiation of the epithelium. So the cysts with a well-differentiated epithelium (RC and DC) express CK-7, while the cysts with a less well-differentiated epithelium (OKC) show slight positivity. Thus it can be useful to differentiate OKC from DC and RC.

Keywords: cytokeratin-7, immunohistochemistry, dentigerous cysts, radicular cysts, odontogenic keratocysts

\section{Introduction}

Odontogenic cysts (OCs) are among the most common destructive lesions affecting the jaw bones [1]. According to different studies, 7 to $12 \%$ of oral and maxillofacial biopsies are odontogenic cysts [2]. The epithelial lining of the odontogenic cysts is derived from the three kinds of epithelium of tooth forming organ: the epithelial rests of Malassez, the reduced enamel epithelium, the epithelial rests or glands of Serres. The potential for further differentiation and proliferation of these epithelial cells during formation of a cyst may differ and lead to differences in epithelial expression and biological behavior among different cyst types [3]. Radicular cysts are the most common cysts of the jaws followed by dentigerous and the odontogenic keratocysts. Ochsenius et al. in 2007 evaluated frequency of odontogenic cysts, Among these radicular cysts accounts for $50.7 \%$, dentigerous cysts for $18.5 \%$ and odontogenic keratocysts account for only $14.3 \%$ [4].

Radicular cyst arises from the epithelial residues (rests of Malassez) in the periodontal ligament as a consequence of inflammation, usually following the death of the dental pulp. This cyst usually associated with apices of non 
vital tooth. The dentigerous cyst encloses the crown and is attached to the neck of an unerupted tooth and develops by accumulation of fluid between the reduced enamel epithelium and the crown or between the layers of the reduced enamel epithelium. The odontogenic keratocyst occurs in the tooth bearing areas of the jaws, or posterior to the mandibular third molar and is derived from the remnants of embryonic dental lamina $[5,6]$.

Cytokeratins are the main structural proteins in epithelial cells representing a class of intermediate filaments. Cytokeratins are grouped as acidic and basic subfamily. Cytokeratin patterns differ in the types of oral epithelium. The expression of the individual CKs is strongly correlated with the pathway of differentiation of cells and tissues. From specific CK expression, conclusions can be drawn about the origin, morphologic characteristics, and function of epithelia confirming a pathological diagnosis or rather the origin of pathology [7].

CK7 is a type II intermediate filament protein with an approximate molecular weight of 54-kD and is expressed in simple epithelia, trachea, transitional epithelium, several complex glands. Basically CK7 belongs to the neutralbasic type that may be expressed in normal epithelium but not in the cyst with that origin or vice versa [8].

There are a number of studies [9-12] that evaluated the expression of various types of cytokeratins in the epithelial linings of jaw cysts to elucidate the pathogenesis, histology and type of origin of different types of odontogenic cysts and have shown positive expression as well but literature search has revealed that there are few studies [13] that have focused on the expression of cytokeratin-7.

Cytokeratin-7 being a simple epithelium marker if expressed in odontogenic cysts could provide us another clue for the change in the pathogenesis of the various types of odontogenic cysts if any. Therefore, with this aim the present study carried out to evaluate the cytokeratin-7 expression in the epithelial linings of odontogenic cysts by immunohistochemistry to unveil certain queries that could bring some new advances into the limelight.

\section{Methods}

The material for the study included 39 formalinfixed, paraffin embedded tissue blocks retrieved from the Department of Oral and Maxillofacial Pathology. These included 15 cases of dentigerous cyst, 12 cases of radicular cyst, and 12 cases of odontogenic keratocyst (OKC). Eight control specimens including periampullary carcinoma, nasopalatine duct cysts and dental follicle were included for immunohistochemical staining with cytokeratin-7. All these cases were diagnosed by routine hematoxylin and eosin staining and then subjected to immunohistochemical staining with the use of Monoclonal Mouse Anti-Human Cytokeratin-7. Ethical clearance was obtained prior to study from the ethical review board of the institute.

\section{Procedure for immnunohistochemistry}

$4 \mu \mathrm{m}$ thick sections were taken on to Poly-L-Lysine adhesive coated micro slides and incubated in hot air oven at $60^{\circ} \mathrm{C}$ for overnight. The sections were then cleared and hydrated through 3 changes of xylene and alcohol respectively, 5 minutes each and bought to distilled water. Antigen retrieval was carried out in pressure cooker with tris EDTA for 40 minutes. Tissue sections were then washed in tris buffer I for 5 minutes and after that in tris buffer II for 5 minutes. For endogenous peroxidase blocking, slide racks were immersed in 3\% hydrogen peroxide in methanol for 10 minutes. Further the sections were washed in distilled water for 3 minutes.

Subsequently each section was then incubated with Primary Monoclonal Mouse Anti-Human Cytokeratin-7 Antibody for 1 hour. The sections were then washed in tris buffer I and II for 5 minutes each and then Secondary antibody (Envision + HRP) was applied to each section for 30-40 minutes and then again washed in tris buffer I and II for 5 minutes each.

For visualization sections were then treated with diaminobenzidine-hydrogen peroxide as a substrate for the peroxidase for 10 minutes followed by which it was washed in distilled water. The sections were then counterstained with hematoxylin and then mounted with DPX. For reagent control, no primary antibody was added.

\section{Immunohistochemical assessment.}

The semiquantitative analysis of degree and intensity of staining was done.

The entire tissue section was observed for extent of staining. The sections were placed on Olympus CX31Research microscope and the cells were projected onto the monitor via Magnus pro MIPS (Olympus) photomicrograph attachment with camera for photomicrography. At least 4 field in the lining epithelium each containing around 100 epithelial cells or more were observed and counted. The epithelial layers predominantly containing the positive cells and pattern of staining were also noted [14].

The immunoreactivity of cytokeratin 7 was ranked into three groups according to percentage of positively stained cells [15].

1. (-) No staining: $\leq 5 \%$ epithelial cells positive

2. (+) Slight/ moderate staining: $>5 \%$ to $<25 \%$ epithelial cells positive

3. (++) Intense staining: $\geq 25 \%$ epithelial cells positive

To assess intensity of staining, slides were evaluated on the basis of amount of chromogen present and were graded into negative, light, medium and dark staining [7].

\section{Statistical analysis}

The collected data were entered into Microsoft Excel 2007 and subjected to statistical analysis using SPSS version 20.0 (IBM Statistics Inc., Chicago, Illinois, USA). The statistical test used was chi-square test to assess the association between different odontogenic cysts used in this study and Cytokeration- 7 staining. The difference was considered to be of statistical significance if the $p$ value was $\leq 0.05$. 


\section{Results}

The samples of odontogenic cysts included 15 (31.91\%) dentigerous cysts, $12(25.53 \%)$ radicular cysts and $12(25.53 \%)$ odontogenic keratocysts. The samples of 5 (10.63\%) periampullary carcinoma, $2(4.25 \%)$ nasopalatine duct cysts and $1(2.12 \%)$ dental follicle were taken as controls (Figure 1). Thus a total of 47 cases included in the present study were subjected to immunohistochemical staining to study the expression of CK7.

\section{Analysis of $\mathrm{CK} 7$ expression}

When CK7 expression was analyzed in various odontogenic cysts, it was found that the expression varied from one cyst to another mainly on the basis of type of epithelium and based on the type of inflammation present.

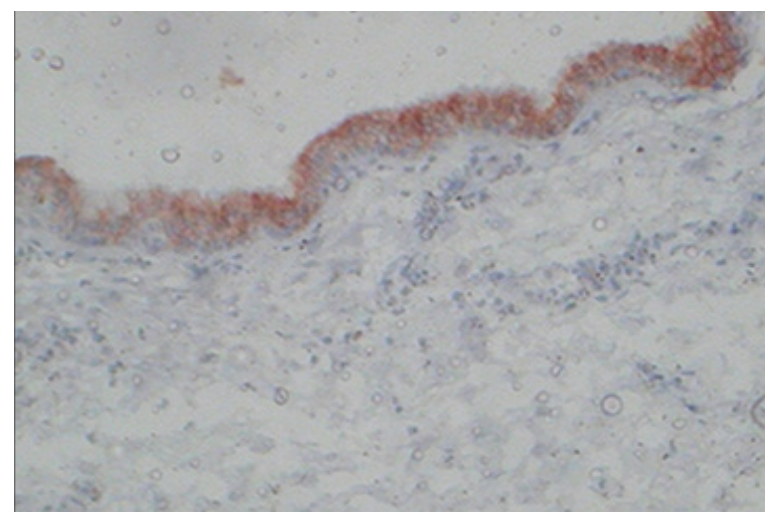

Figure 1. Photomicrograph showing diffuse immunopositive superficial cells for CK7 in the epithelial lining of nasopalatine duct cyst. NPDC was used as a positive control in the present study (20X).

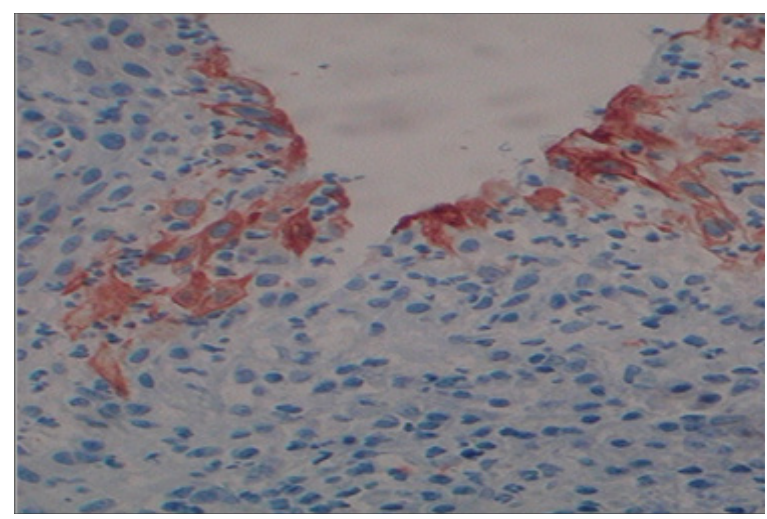

Figure 3. Photomicrograph showing focal cytoplasmic immunopositive staining of CK7 in the superficial cells of radicular cyst lining (20X).
CK7 expression was maximum in DC (66.66\%) (Figure 2) followed by RC (41.66\%) (Figure 3) and OKC (16.6\%) (Figure 4) (Table I). Thus, in the present study, CK7 was discernible in only 2 of 12 cases of odontogenic keratocysts. In contrast, dentigerous cysts showed a positive staining in 10 of 15 cases. This represented a statistically significant difference between dentigerous cysts and odontogenic keratocysts at a probability level of $<0.01$ ( $p$-value $=$ 0.009). There was also a difference in the expression of CK7 in RC and DC, as RC only showed positive expression in 5 of 12 cases but this difference was not found to be statistically significant. The immunopositivity of CK7 was also compared between RC and OKC and the difference was not found to be statistically significant.

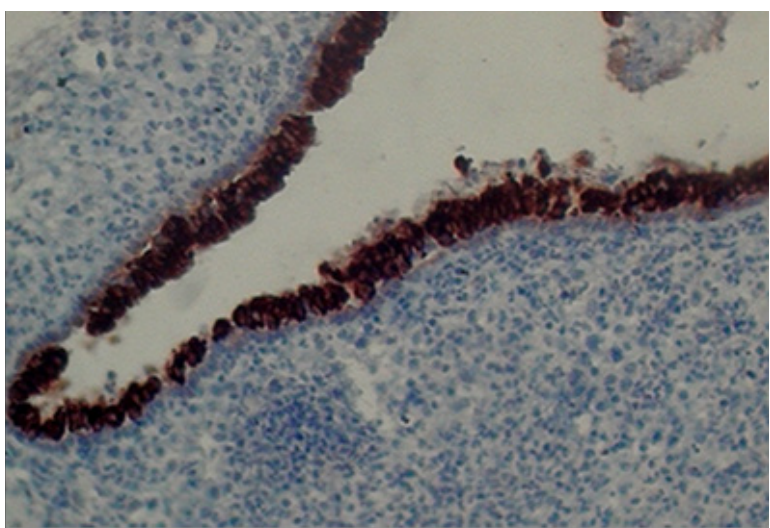

Figure 2. Photomicrograph showing diffuse and dark immunopositive suprabasal cells for CK7 in the epithelial lining of dentigerous cyst (20X).

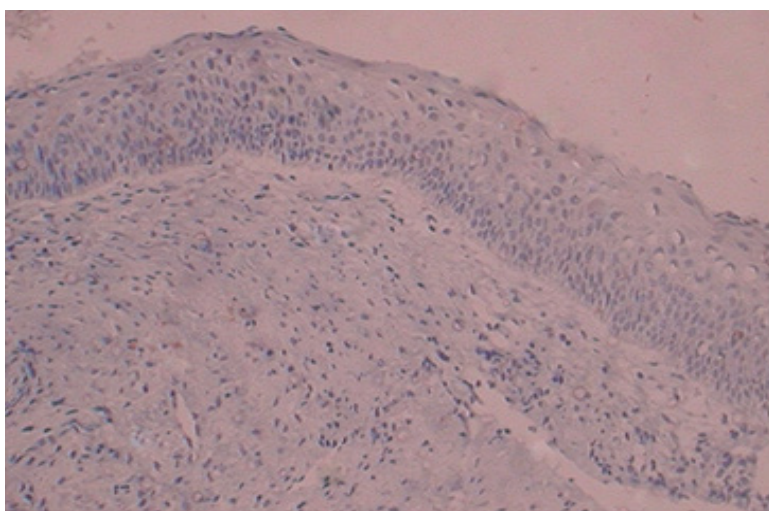

Figure 4. Photomicrograph showing epithelial lining of odontogenic keratocyst without any positive staining for CK7 (20X).

Table I. Quantitative analysis of immunohistochemical findings of CK7 in dentigerous cysts, odontogenic keratocysts and radicular cysts.

\begin{tabular}{l|c|ccc}
\hline Lesion & Total no. of patients & \multicolumn{3}{|c|}{ Immunohistochemical findings } \\
\cline { 4 - 5 } & & - & + & ++ \\
Dentigerous cysts & $15(100 \%)$ & $5(33.33 \%)$ & $4(26.66 \%)$ & $6(40.0 \%)$ \\
Odontogenic keratocysts & $12(100 \%)$ & $10(83.33 \%)$ & $1(8.3 \%)$ & $1(8.3 \%)$ \\
Radicular cysts & $12(100 \%)$ & $7(58.33 \%)$ & $3(25.0 \%)$ & $2(16.66 \%)$
\end{tabular}

RC VS DC: $x^{2}=1.69 ; p$ value $=0.19 ;$ RC VS OKC: $x^{2}=1.82 ; p$ value $=0.17 ;$ DC vs. OKC: $x^{2}=6.75 ; p$ value $=0.009 *$; Chi square test; ${ }^{*} \mathrm{p}<0.05$, Significant 
Table II. Expression pattern of CK7 in odontogenic cysts.

\begin{tabular}{l|c|c|c|c|c|}
\multirow{2}{*}{ Lesion } & & & \multicolumn{3}{|c}{ Layer involved } \\
\cline { 2 - 6 } DC & Total & Positive & Superficial & Suprabasal & Spinous \\
OKC & 15 & $10(66.66 \%)$ & $4(40 \%)$ & $6(60 \%)$ & 0 \\
RC & 12 & $2(16.66 \%)$ & $1(50 \%)$ & 0 & $1(50 \%)$ \\
& 12 & $5(41.66 \%)$ & $3(60 \%)$ & 0 & $2(40 \%)$
\end{tabular}

RC VS DC: $\mathrm{x}^{2}=4.29 ; \mathrm{p}$ Value $=0.12$; RC VS OKC: $\mathrm{x}^{2}=0.38 ; \mathrm{p}$ Value $=0.54 ;$ DC VS OKC: $\mathrm{x}^{2}=5 ; \mathrm{p}$ Value $=0.08$; Chi square test used

Table III. Staining pattern of CK7 in odontogenic cysts.

\begin{tabular}{l|c|c|c|c|}
\multirow{2}{*}{ Lesion } & \multirow{2}{*}{ Total } & \multirow{2}{*}{ Positive } & \multicolumn{2}{|c}{ Staining pattern } \\
\cline { 4 - 5 } & & & Focal & Uniform \\
\hline DC & 15 & $10(66.66 \%)$ & $4(40 \%)$ & $6(60 \%)$ \\
OKC & 12 & $2(16.66 \%)$ & $1(50 \%)$ & $1(50 \%)$ \\
RC & 12 & $5(41.66 \%)$ & $5(100 \%)$ & 0
\end{tabular}

RC vs DC: $\mathrm{x}^{2}=5.0, \mathrm{p}$-value $=0.025^{*} ; \mathrm{DC}$ vs OKC: $\mathrm{x}^{2}=0.27, \mathrm{p}$-value $=0.6 ; \mathrm{RC}$ vs OKC: $\mathrm{x}^{2}=2.92, \mathrm{p}$-value $=0.08$ Chi square test; * $\mathrm{p}<0.05$, Significant

Table IV. Correlation of intensity of staining of CK7 in odontogenic cysts.

\begin{tabular}{|c|c|c|c|c|c|c|}
\hline \multirow{2}{*}{ Lesion } & \multirow{2}{*}{ Total } & \multicolumn{4}{|c|}{ Intensity } & \multirow{2}{*}{ P value } \\
\hline & & Nil & Low & Intermediate & High & \\
\hline DC & 15 & $5(33.33 \%)$ & $4(26.66 \%)$ & $1(6.66 \%)$ & $5(33.33 \%)$ & 0.28 \\
\hline OKC & 12 & $10(83.33 \%)$ & $1(8.33 \%)$ & $1(8.33 \%)$ & 0 & $<0.001 * *$ \\
\hline RC & 12 & $7(58.33 \%)$ & $4(33.33 \%)$ & 0 & $1(8.33 \%)$ & $0.003 * *$ \\
\hline
\end{tabular}

RC vs DC: $\mathrm{x}^{2}=3.71, \mathrm{p}$-value $=0.29 ;$ DC vs OKC: $\mathrm{x}^{2}=8.24, \mathrm{p}$-value $=0.041 *$ RC vs OKC: $\mathrm{x}^{2}=4.33$, $\mathrm{p}$-value $=0.23$ Chi square test; ${ }^{*} p<0.05$, Significant

\section{Staining and expression pattern}

The staining and expression pattern was different for different cysts. DC showed uniform expression in $60 \%$ cases and was discernible suprabasally in almost all of the cases (Figure 2).When the layers involved in different cysts were compared regarding CK 7 expression, the results were nonsignificant. But their staining pattern was different and was statistically significant with a $\mathrm{p}$-value $=0.025$ (between DC and RC). DC showed uniform pattern in $60 \%$ cases whereas not even a single case of RC showed uniform pattern of expression (Table II, III). Regarding staining intensity 5 different observers individually analyzed the slides. In cases, where scores differed, the majority decision was adopted. In case of DC, 5 cases showed high staining intensity whereas none of RC showed such an expression. Thus when the staining intensity was compared between different groups the results came out to be statistically significant in case of DC and OKC with p-value $=0.041$ (Table IV).

\section{Discussion}

Odontogenic cysts form an important spectrum of oral diseases which occur commonly and are often destructive to maxillofacial apparatus. Epithelial linings of various odontogenic cysts histologically may appear similar in areas of inflammation. Therefore it is often important to determine the site of origin because this may affect the choice of treatment $[16,17]$.

Literature indicates that keratin expression in the epithelial linings of the odontogenic cysts correlates with histologic differentiation [13]. From specific Cytokeratin expression, conclusions can be drawn about the origin, morphologic characteristics, and function of epithelia. The immunohistochemical detection of different cytokeratins polypeptides has made it easier to explain the histogenesis of many epithelial diseases [18].

CK7 belongs to the neutral-basic type subfamily with an approximate molecular weight of 54-kd and its anatomic distribution is generally restricted to epithelia and their neoplasms [19]. During cell transformation and tumor development, the cell type specificity of CK7 is largely conserved [14]. Thus, the present study was carried out to elucidate the expression of Cytokeratin-7 in epithelial linings of various odontogenic cysts.

\section{CK7 expression, staining and expression pattern}

\section{in radicular cysts}

In the present study, 12 specimens of radicular cysts were taken and out of those 12 cases CK7 showed immunopositivity in only 5 cases. The results were 
in accordance with the previous studies of Hormia et al. (1987) [20], Gao et al. (1988) [21] and Stoll et al. (2005) [14]. In our study $41.66 \%$ radicular cysts showed immunopositivity for CK7 whereas in the study conducted by Stoll et al. $26.7 \%$ and in the study by Gao et al. $40 \%$ of RC showed CK7 immunopositive cells. Radicular cysts in the present study showed patchy expression of CK7 in all the cases and positive staining was restricted to the superficial cell layers. This was in accordance with the study of Stoll et al. (2005) [14]. In the study of Hormia et al. (1987) [20] all layers of radicular cysts have been shown to be positive with an antibody reacting with CK 7, 17 and 19 whereas in another study of Matthews et al. (1988) [13] antibody specific for CK7, only one of 25 radicular cysts was positive. The variation in the staining pattern may occur depending upon the type of antibody used. In some instances, lining epithelium of radicular cyst is derived from paranasal respiratory epithelium, and that squamous metaplastic sequence may follow by maintaining the same keratin (CK7) expression. As a result, its outer layer cells which were perhaps originally columnar may stain positive with the CK7 antibody while newly formed deeper layer cells do not possess this cytokeratin [22]. So, this might be the reason for the expression of CK7 in the superficial cell layers of radicular cysts in the present study.

CK7 expression, staining and expression pattern in dentigerous cysts

When CK7 expression was evaluated in dentigerous cysts, it was discernible in 10 out of 15 cases making immunopositivity of $66.66 \%$. When the results were compared with the previous studies they were in accordance with the studies of Stoll et al. (2005) [14] where the CK7 was discernible in $76.66 \%$ cases. The results were also similar to the studies of Matthews et al. (1988) [13], Gao et al. (1989) [21], Fregnani et al. (2003) [23].

In this study dentigerous cysts showed the maximum positive immunoexpression for CK7 antibody $(66.66 \%)$ and also showed the uniform expression in $60 \%$ of the cases. Though the staining was restricted to suprabasal and the superficial cell layers in case of dentigerous cysts but the staining intensity and the staining pattern was definitely better than that was seen in case of radicular cysts. This was in accordance with the previous studies of Stoll et al (2005) [14] where $76.66 \%$ of dentigerous cysts stained positive for CK7 antibody. The result was contradictory when it was compared to the study of Hormia et al (1987) [20] where all layers of dentigerous cysts showed positive immunoexpression for CK7 antibody. This could have occurred because in their study antibody specific for CK7 was not used.

CK7 expression, staining and expression pattern in odontogenic keratocysts

In the present study, 12 specimens of odontogenic keratocysts were taken and CK7 was discernible in only $2(16.6 \%)$ cases. out of these 1 had very good and 1 had diffuse expression of CK7. These results were slightly different from the previous studies of Stoll et al. (2005) where OKCs showed immunopositivity for CK7 antibody in $60 \%$ cases. Also in the study of Hormia et al (1987) [20] and Domingues et al. (2000) [24] CK7 was positive in most of the OKCs. According to Marx et al (2003) [25] OKC may also arise from reduced enamel epithelium which may change the keratin profile. In this 1 case, there might be a possibility that the cyst arose from the source other than the remnants of dental lamina and thus produced such a diffuse expression. In our study the staining was found to be was restricted to the suprabasal cell layers. The results of the study conducted by Stoll et al. (2005) [14] were similar to ours as in their study also the staining was restricted to suprabasal cell layers only in case of OKCs and basal layer was left blank in each case.

Comparison of $C K 7$ expression between radicular cysts, dentigerous cysts and odontogenic keratocysts

When the comparison was made between the three cysts namely radicular, dentigerous and odontogenic keratocysts, it was revealed that there was a significant difference in the expression of CK7 antibody in between dentigerous cysts and odontogenic keratocysts. CK7 was discernible in only 2 of $12 \mathrm{OKCs}(16.66 \%)$. In contrast dentigerous cysts showed a positive staining in $66.66 \%$ cases. Thus in the present study the antibody OV-TL 12/30 represented a statistically significant difference between $\mathrm{OKC}$ and dentigerous cysts at a probability level of $<0.01$. Though radicular cysts showed positive staining in $41.66 \%$ cases only but the difference was not statistically significant. This was slightly different from the study of Stoll C et al. (2005) [14] where they found positive staining in $40 \%$ radicular cysts, $76.66 \%$ dentigerous cysts and $60 \%$ odontogenic keratocysts. This represented a statistically significant difference between radicular and dentigerous cysts at a probability level of $<0.01$ which was not seen in the present study. Other studies have revealed results comparable with ours, namely a part of $37 \%$ positive radicular cysts, $59 \%$ positive dentigerous cysts, and $29 \%$ positive odontogenic keratocysts [26] or $70 \%$ positive dentigerous cysts and $21 \%$ positive odontogenic keratocysts [27].

\section{Conclusion}

In the present study dentigerous cysts showed the maximum immunopositive expression for CK7. It was followed by radicular cysts and odontogenic keratocysts respectively. Dentigerous cysts showed the uniform epithelial expression of CK7 in maximum cases whereas radicular cysts showed the patchy expression in all the immunopositive cases. Odontogenic keratocysts showed positive immunoexpression in only two cases with patchy expression in one and uniform in the other. Hence, we conclude that the immunoexpression of CK7 in the epithelial lining of odontogenic cysts depends on the source 
of origin and differentiation. Its expression correlates with the degree of differentiation of the epithelium. So the cysts with a well-differentiated epithelium (RC and DC) express CK-7, while the cysts with a less well-differentiated epithelium (OKC) show slight positivity. Thus it can be useful to differentiate OKC from DC and RC, this marker CK7 being easily available and cost effective for the diagnosis of various body tumors can be used in future for studying the pathogenesis of odontogenic cysts instead of the higher priced markers. Further studies with an increased sample size and using various epithelial and mesenchymal markers and antibodies should be carried out to confirm the effect of source of origin on pathogenesis and epithelial differentiation of various odontogenic cysts.

\section{References}

1. Avelar RL, Antunes AA, Carvalho R, Bezerra P, Oliveira Neto PJ, Andrade ES. Odontogenic cysts: a clinicopathological study of 507 cases. J Oral Sci. 2009;51:581-586.

2. Mosqueda-Taylor A, Irigoyen-Camacho ME, Diaz-Franco MA, Torres-Tejaro MA. Odontogenic cysts. Analysis of 856 cases. Med Oral. 2002;7:89-96.

3. Tie J, Kitano M. Epithelial cell proliferation in the odontogenic keratocysts: a review. Ann Kagoshima University Dental School. 1997;17:27-40.

4. Ochsenius G, Escobar E, Godoy L, Peñafiel C. Odontogenic cysts: analysis of 2,944 cases in Chile. Med Oral Patol Oral Cir Bucal. 2007;12:E85-E91.

5. Shear M, Speight PM. Cysts of the oral and maxillofacial regions. 4th ed. Blackwell Munksgaard, 2007; pp 6-142.

6. Shafer WG, Hine MK, Levy BM. A Textbook of Oral Pathology. 4th ed. Saunders, 2004; pp 258-273.

7. Schwerer MJ, Kraft K, Baczako K, Maier H. Coexpression of cytokeratins typical for columnar and squamous differentiation in sinonasal inverted papillomas. Am J Clin Pathol. 2001;115:747-754.

8. Moll R, Franke WW, Schiller DL, Geiger B, Krepler R. The catalog of human cytokeratins: patterns of expression in normal epithelia, tumors and cultured cells. Cell. 1982;31:11-24.

9. Kathuri D, Kattappagari KK, Pusarla C, Teja R, Gontu SR, Ramana Reddy BV. Quantitative analysis of cytokeratin 19 expression in Odontogenic Keratocyst, Dentigerous cyst and Radicular Cyst. Indian J Appl Res. 2015;5:433-435.

10. Deepa K, Munisekhar MS, Suri C, Rajalbandi SK, Praveen MR, Gothe P. Comparison of immunohistochemical expression of CD10 in odontogenic cysts. J Clin Diag Res 2014;8:ZC35-ZC38.

11. Ramadoss R, Krishnan R, Peddanna SK, Cherian E, Gunasekaran N, Thayalan D. Immunohistochemical analysis of cytokeratin 10, cytokeratin 14, epidermal growth factor receptor, tenascin, and Ki-67 in selected odontogenic cysts. SRM J Res Dent Sci. 2015;6:96-100.

12. Yarlagadda K, Kamath VV, Satelur K. Immunohistochemical expression of keratins 8 and 19 in odontogenic cysts and tumors. J Cranio Max Dis. 2015;4:128-136.

13. Matthews JB, Mason GI, Browne RM. Epithelial cell markers and proliferating cells in odontogenic jaw cysts. J Pathol. 1988;156:283-290.

14. Stoll C, Stollenwerk C, Riediger D, Mittermayer C, Alfer J. Cytokeratin expression patterns for distinction of odontogenic keratocysts from dentigerous and radicular cysts. J Oral Pathol Med. 2005;34:558-564.

15. Suzuki T, Kumamoto H, Kunimori K, Ooya K. Immunohistochemical analysis of apoptosis-related factors in lining epithelium of radicular cysts. J Oral Pathol Med. 2005;34:46-52.

16. Açikgöz A, Uzun-Bulut E, Özden B, Gündüz K. Prevalence and distribution of odontogenic and nonodontogenic cysts in a Turkish population. Med Oral Patol Oral Cir Bucal. 2012;17:e108-e115.

17. Ramachandra P, Maligi P, Raghuveer H. A cumulative analysis of odontogenic cysts from major dental institutions of Bangalore city: A study of 252 cases. J Oral Maxillofac Pathol. 2011;15:1-5.

18. Crivelini MM, de Araújo VC, de Sousa SO, de Araújo NS. Cytokeratins in epithelia of odontogenic neoplasms. Oral Dis. 2003;9:1-6.

19. Chu P, Wu E, Weiss LM. Cytokeratin 7 and cytokeratin 20 expression in epithelial neoplasms: a survey of 435 cases. Mod Pathol. 2000;13:962-972.

20. Hormia M, Ylipaavalniemi P, Nagle RB, Virtanen I. Expression of cytokeratins in odontogenic jaw cysts: monoclonal antibodies reveal distinct variation between different cyst types. J Oral Pathol. 1987;16:338-346.

21. Gao Z, Mackenzie IC, Williams DM, Cruchley AT, Leigh I, Lane EB. Patterns of keratin-expression in rests of Malassez and periapical lesions. J Oral Pathol. 1988;17:178-185.

22. Maeda Y, Hirota J, Yoneda K, Osaki T. Immunohistochemical study of jaw cysts: different existence of keratins in odontogenic and non-odontogenic epithelial linings. J Oral Pathol Med. 1990;19:289-294.

23. Fregnani ER, Pires FR, Quezada RD, Shih leM, Vargas PA, de Almeida OP. Calcifying odontogenic cyst: a clinicopathological features and immunohistochemical profile of 10 cases. J Oral Pathol Med. 2003;32:163-170.

24. Domingues MG, Jaeger MM, Araújo VC, Araújo NS. Expression of cytokeratins in human enamel organ. Eur J Oral Sci. 2000;108:43-47.

25. Marx RE, Stern D. Oral and Maxillofacial Pathology: A rationale for Diagnosis and Treatment. Quintessence Publication, 2003; pp 579-601.

26. Wagner Y, Filippi A, Kirschner H, Dreyer T. Cytokeratin and p53 expression of odontogenic cysts. Mund Kiefer Gesichtschir. 1999;3:263-269.

27. Pires FR, Chen SY, da Cruz Perez DE, de Almeida OP, Kowalski LP. Cytokeratin expression in central mucoepidermoid carcinoma and glandular odontogenic cyst. Oral Oncol. 2004;40:545-551. 